\title{
An evolutive descriptive mapping visualisation tool with the integrated GAIA-AHP
}

\author{
Alessio Ishizaka ${ }^{1}$, Sajid Siraj ${ }^{1,2,3}$, Phillipe Nemery ${ }^{4}$ \\ ${ }^{1}$ Centre for Operational Research and Logistics, Portsmouth Business \\ School, University of Portsmouth, Portsmouth PO1 3DE, United \\ Kingdom \\ Alessio.Ishizaka@port.ac.uk \\ ${ }^{2}$ COMSATS Institute of Information Technology, The Mall, Wah \\ Cantonment, Pakistan \\ ${ }^{3}$ Centre for Decision Research, Leeds University Business School, \\ Leeds, United Kingdom \\ sajidsiraj@gmail.com \\ ${ }^{4}$ SAP BeLux, Avenue des Olympiades 2, 1140 Brussels, Belgium \\ pnemery@gmail.com
}

\begin{abstract}
Although Multi-Criteria Decision Making methods have been extensively used, their descriptive use has been rarely considered. In this paper, we add an evolutionary description phase as an extension to the AHP method that helps policy makers to gain insights into their decision problems. The proposed extension has been implemented in an open-source software that allows the users to visualise the difference of opinions within a decision process, and also the evolution of preferences over time. The method was tested in a two-phase experiment to understand the evolution of opinions on energy sources. The proposed tool can help policy makers in better understanding planning problems that will lead towards better decisions.
\end{abstract}

Keywords: Multi Criteria Decision making; Group judgments; AHP; GAIA; Visualization.

\section{Introduction}

Multi-criteria decision making (MCDM) methods have been extensively used to prioritize available options after assessing and synthesizing all the individual preferences. However, the aggregative approaches like Analytic Hierarchy Process (AHP) have low explanatory power and results are often not enough to reach to a consensual decision by stakeholders, especially when they have divergent views [1]. It is therefore necessary to identify the points of agreements and disagreements before initiating a negotiation process. In response to this need, Graphical Analysis for Interactive Aid (GAIA) [2] was developed to capture 
different views of DMs with respect to many criteria and to display them graphically. The GAIA method was initially proposed to complement PROMETHEE which is widely used in strategic decision making [ㄹ]

\section{Research design,methodology and results}

In this paper, we propose to combine the GAIA method with AHP and show its usefulness in the area of energy policy making []ㅡ. We investigate the visualization of preferences and their evolution in situations where additional information is acquired during the decision making process. The proposed combination (AHP-GAIA) displays a graphical representation that can easily highlight the presence of any like-minded decision makers/agents or opposite minds, and can also reflect changes in their preferences over time. This hybrid method has been applied in a two-phase experiment. In the first phase, participants were asked to rank seven energy sources in the United Kingdom for the next 20 years. Each participant compared the options in pairs without any specific tool. For the second phase, the participants were informed about the widely-used criteria to evaluate the energy sources, and were asked to produce a documented report on how well the energy sources were fulfilling these criteria. Three months later, the participants submitted their reports that showed their analyses of the seven energy sources with the help of AHP. The adapted GAIA approach was then used to visualize the change in participants' preferences. In both phases, the solar energy has been found to be the most preferred choice, while coal remained the least preferred (figure 1). Interestingly, the dispersion of the opinions decreased in the second phase i.e. more participants were found in agreement with each other after performing the detailed analysis of the selected energy sources.

\section{References}

1. Buchholz, T., et al., Multi Criteria Analysis for bioenergy systems assessments. Energy Policy, 2009. 37(2): p. 484-495.

2. Brans, J.-P. and B. Mareschal, The PROMCALC \& GAIA decision support system for multicriteria decision aid. Decision Support Systems, 1994. 12(4-5): p. 297-310.

3. Ishizaka, A., S. Siraj, and P. Nemery, Which energy mix for the UK? An evolutive descriptive mapping with the integrated GAIA-AHP visualisation tool. Energy, 2016: p. advance online publication, doi: 10.1016/j.energy.2015.12.009. 
Figure 1: Visualizing the alternatives and decision makers in PriEsT for the intuitive approach

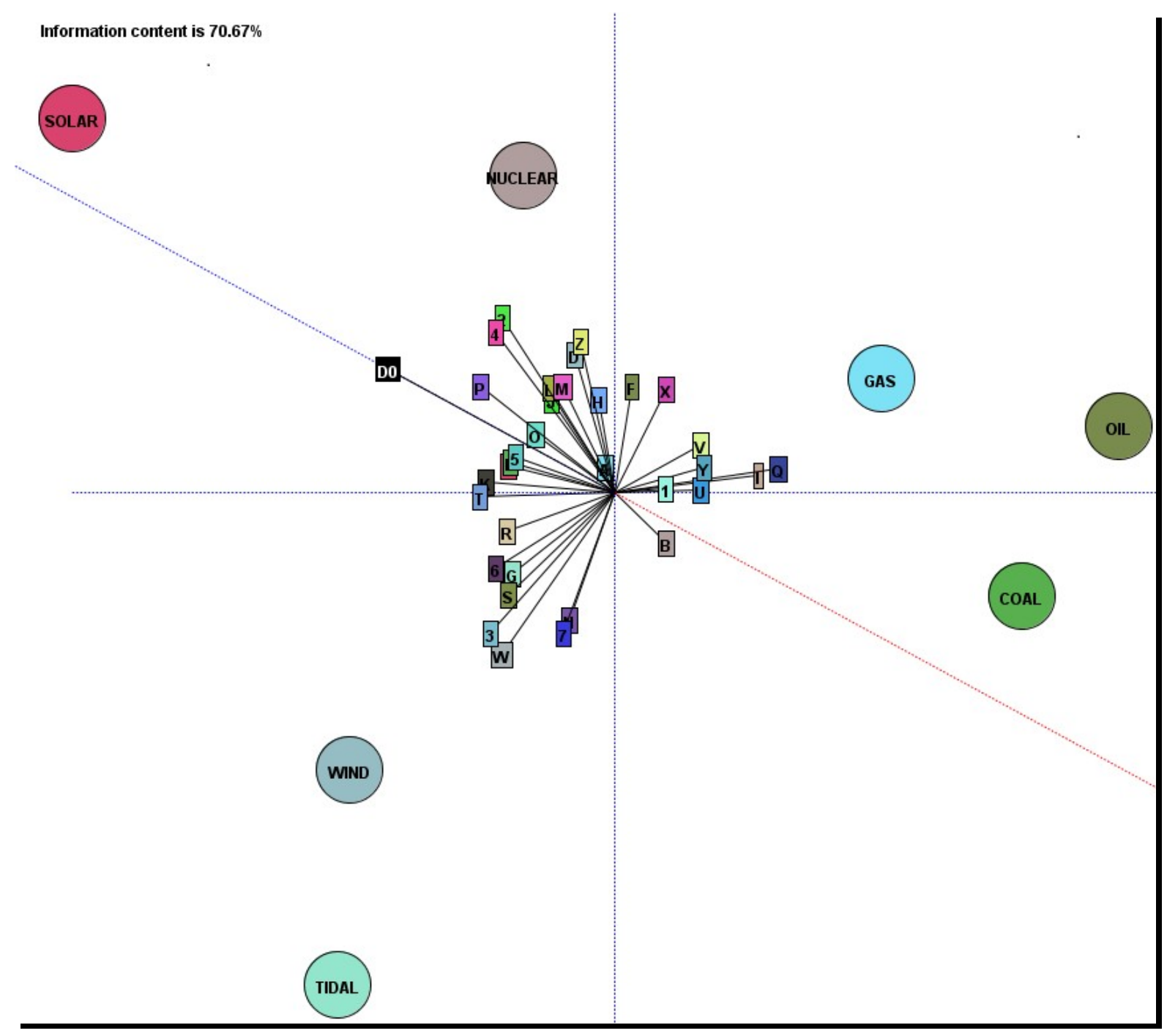

